The mean pre-ductal saturations were lower than post-ductal saturations in all groups except the group not having a PDA and not on respiratory support (figure 1).

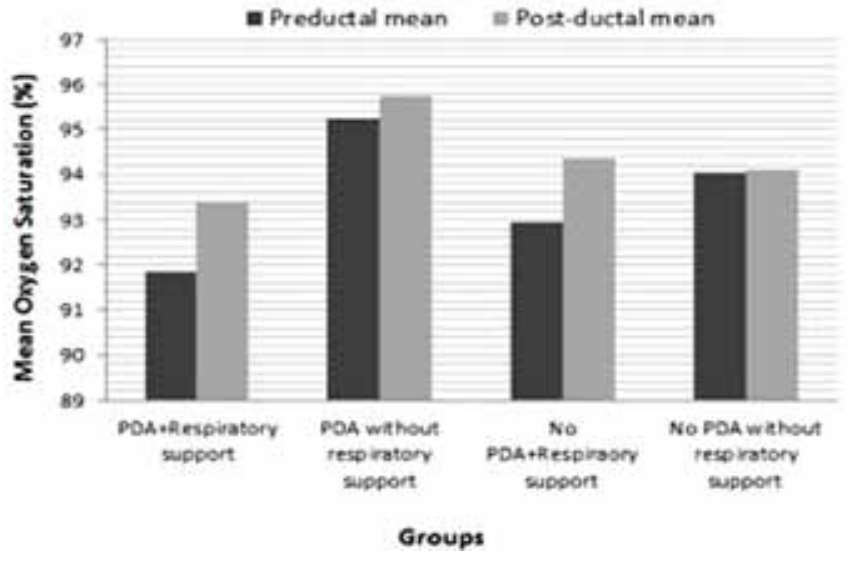

Abstract 1794 Figure 1

Conclusions The site of measurement can affect the observed oxygen saturation values and hence should be taken in account while bedside monitoring and planning clinical trials.

\section{DID VENTILATORY STRATEGY CHANGE DURING THE LAST 5 YEARS IN ITALIAN NEONATAL NETWORK?}

doi:10.1136/archdischild-2012-302724.1795

${ }^{1} \mathrm{~V}$ Vendettuoli, ${ }^{2} \mathrm{M}$ Condò, ${ }^{3} \mathrm{~A}$ Poloniato, ${ }^{4} \mathrm{M}$ Raia, ${ }^{5} \mathrm{~F}$ Ramacciato, ${ }^{6} \mathrm{LG}$ Tina, ${ }^{7} \mathrm{LM}$ Abbiati, ${ }^{8} \mathrm{~A}$ Staffler, ${ }^{9} \mathrm{~S}$ Agostiniani. 'NICU Fondazione IRCCS Ca' Granda Ospedale Maggiore Policlinico - Università degli Studi di Milano, Milan; ${ }^{2}$ Division of Neonatology and NICU, Ospedale A. Manzoni, Lecco; ${ }^{3}$ Unità Operativa di Neonatologia e Patologia Neonatale Dipartimento Materno-Infantile - H San Raffaele, Milan; ${ }^{4}$ SCDU Neonatologia ASO OIRM S. Anna Università di Torino, Torino; ${ }^{5}$ Division of Neonatology and NICU Ospedale Cardarelli, Campobasso; ${ }^{6}$ TIN ARNAS Ospedale Garibaldi, Catania; ${ }^{7}$ U.O. Neonatologia e Terapia Intensiva Neonatale - Fondazione MBBM - Ospedale San Gerardo Monza, Monza; ${ }^{8}$ Department of Neonatology, Regional Hospital of Bolzano, Bolzano; ${ }^{9} \mathrm{AOU}$ MEYER, Firenze, Italy

Background Intubation and mechanical ventilation (MV) are lifesaving procedures but are associated with a higher incidence of acute and chronic complications. Thus, non-invasive ventilation (NIV: nasal continuous pulmonary distending pressure, nasal ventilation, or high-flow nasal cannula) is increasingly used.

Aim To evaluate changes in ventilatory strategies between 2006 and 2010 in Italian neonatal network (INN).

Methods A cohort of neonates $<30$ weeks gestational age (GA) or $<1501 \mathrm{~g}$ birth weight (BW), without congenital anomalies, born in 2006 and 2010, assisted in 31 hospitals participating in INN both years, was analysed ( $N=3459$ : 1713 in 2006, and 1746 in 2010). Variables were defined according to Vermont-Oxford network. Logistic regressions, adjusting for confounders (GA, BW for GA, antenatal steroids, mode of delivery, multiple pregnancy, 1-minute Apgar score, being inborn, sex, intubation in delivery room, RDS, PDA), and clustering for hospitals, were used.

Results Between 2006 and 2010 there were no changes in GA or BW (2006: mean GA 29.1 wks; BW 1087 g; 2010: GA 29.2 wks; BW $1083 \mathrm{~g}$ ), while antenatal steroids increased (from $78.5 \%$ to $83.5 \%$ ). The number of infants receiving any ventilatory support increased from $81.8 \%$ to $85.9 \%$. After adjusting for confounders, mortality decreased (Odds ratio $=0.75,95 \%$ confidence interval $0.57-0.98$ ) as well as mechanical ventilation $(\mathrm{OR}=0.72,95 \% \mathrm{CI} 0.57-0.90)$ and $\mathrm{BPD}(\mathrm{OR}=0.68,95 \% \mathrm{CI}$ 0.54-0.86), while NIV increased $(\mathrm{OR}=1.70$, $95 \%$ CI 1.41-2.04).
Conclusions In the last 5 years, we observed a reduction of MV and an increase of NIV use. This was accompanied by a decrease in risk-adjusted mortality and $\mathrm{BPD}$.

\section{INCIDENCE OF AND RISK FACTORS FOR AIR LEAKS IN PRETERM INFANTS EXPOSED TO RESTRICTIVE USE OF ENDOTRACHEAL INTUBATION}

doi:10.1136/archdischild-2012-302724.1796

${ }^{1} \mathrm{H}$ Hummler, ${ }^{1} \mathrm{E}$ Parys, ${ }^{1} \mathrm{~J}$ Essers, ${ }^{1} \mathrm{R}$ Hopfner, ${ }^{1} \mathrm{O}$ Beringer, ${ }^{2} \mathrm{~B}$ Mayer, ${ }^{1} \mathrm{H}$ Fuchs, ${ }^{1} \mathrm{M}$ Schmid. ${ }^{1}$ Dept. of Pediatrics, Children's Hospital, University of Ulm; ${ }^{2}$ Institute of Epidemiology and Medical Biometry, University of UIm, UIm, Germany

Introduction The occurrence of air leaks such as pneumothorax (PTX), pneumopericardium (PPC) and pulmonary interstitial emphysema (PIE) may be a life-threatening condition in preterm infants.

Aim of the Study To study the incidence of and risk factors for air leaks in preterm infants treated with a policy of sustained inflations followed by non-invasive ventilation in the delivery room.

Methods Perinatal variables, variables of delivery room support and respiratory support in the NICU were analyzed retrospectively for infants with/without air leaks in preterm infants $<30$ wks GA born 2005-2009 ( $n=297$ )

Results Median (range) gestational age was $26+0(22+4-29+1)$ wks, birth weight was 790 (265-1660) g and 270/297 (91.0\%) survived. 63 (21.2\%) developed any air leak, 32 (10.8\%) developed PTX, $44(14.8 \%)$ PIE, and $1(0.3 \%)$ PPC. Infants with air leaks had a higher risk for death $(18(28.6 \%)$ vs. 9 (3.8\%), p<0.01) and for IVH Grade 3-4 (16 (25.4\%) vs. 29 (12.4\%), p<0.05). Air Leaks were associated with less use of prenatal steroids (44 (69.8\%) vs. 199 (85.4\%), $\mathrm{p}<0.01)$ and a more common use of cardiac compressions $(9(14.3 \%)$ vs. $11(4.7 \%), \mathrm{p}<0.01)$, use of a pressure of $30 \mathrm{cmH}_{2} \mathrm{O}$ for sustained inflations (32 (55.2)\% vs. $80(36.7 \%), \mathrm{p}<0.05)$ and intubation during initial resuscitation (34 (54.0\%) vs. 60 (25.6\%), p<0.01)

Conclusion Air leaks were associated with an increased risk for mortality and severe IVH. Our approach resulted in a high rate of survival but was associated with a substantial rate of air leaks. Randomized trials are necessary further improve delivery room care.

\section{DO NON-INVASIVE VENTILATORY STRATEGIES WORK IN MICRO-PREMATURE INFANTS WHO ARE AT THE LIMITS OF VIABILITY?}

doi:10.1136/archdischild-2012-302724.1797

E Okulu, S Arsan, IM Akin, S Alan, A Kılıç, B Atasay. Department of Pediatrics, Division of Neonatology, Ankara University, Ankara, Turkey

Aim To evaluate the non-invasive ventilatory support in micropremature infants who are at the limits of viability.

Methods This prospective cohort study from January-2009 to December 2011 included infants born before 26 weeks'. During resuscitation, stabilisation and transport infants were ventilated with a T-piece resuscitator, and all received prophylactic surfactant at a dose of $100 \mathrm{mg} / \mathrm{kg}$. If respiratory drive was present, infants were extubated to NCPAP. The demographic and clinical features of the infants were assessed.

Results Twenty-four infants born during the study period. Antenatal steroid rate was $16.7 \%$. Mean gestational age(GA) and birth weight(BW) were $24.3 \pm 0.9$ weeks, and $660.2 \pm 125.5 \mathrm{~g}$, respectively. The presence of premature rupture of membranes and chorioamnionitis rate was $54 \%$. Only five $(21 \%)$ of 24 infants could be extubated to NCPAP, and three of these five were intubated in first 3-days. Only two(8.3\%) infants succeeded on NCPAP, and the GAs' were 24.6 and 25.1 weeks, the BWs' were 1010 and $730 \mathrm{~g}$. The rate of NEC, PDA, İVH and pulmonary hemorrhage were 29\%, 36\%, 36\% and $21 \%$, respectively in infants who survived more than 2 days. 
The overall mortality rate was $92 \%$, the duration of hospitalization was between one and 137 days.

Conclusion In our study, it has been seen that NCPAP may not be an effective ventilation strategy in premature infants who are at the limits of viability. The high proportion of chorioamnionitis in this group may affect the ventilation and the following problems. These babies are needed to be care at very special settings.

\section{CLINICAL OUTCOMES OF VERY-LOW-BIRTH-WEIGHT INFANTS WHO RECEIVE NON-INVASIVE VENTILATORY SUPPORT}

doi:10.1136/archdischild-2012-302724.1798

E Okulu, S Arsan, IM Akin, S Alan, A Kilıc, B Atasay. Department of Pediatrics, Division of Neonatology, Ankara University, Ankara, Turkey

Aim To evaluate the clinical outcomes of very-low-birth-weight infants received non-invasive ventilation at delivery room (DR) and NICU.

Methods This prospective cohort study included infants born before 29 weeks', and infants born at 29-30 weeks' who didn't receive antenatal steroid (ANS) from January-2009 to December 2011. During resuscitation, stabilization and transport infants were ventilated with a T-piece. All received $100 \mathrm{mg} / \mathrm{kg}$ surfactant. If respiratory drive was present, infants were extubated to nasal CPAP(NCPAP). The need for MV within the first 3-days, neonatal morbidities, mortality, and duration of hospitalization were assessed.

Results Eighty infants were evaluated. Mean gestational age(GA) and the mean birth weight(BW) of infants were 27.0 \pm 2.1 weeks, and $936.5 \pm 299.1 \mathrm{~g}$, respectively. ANS was given to $27.5 \%$ of the pregnancies. The presence of PPROM and chorioamnionitis were $57.5 \%$ and $34 \%$, respectively. Twenty-three (28.7\%) infants could not be extubated at the DR, and mean GA and BW were lower than the infants who could extubated. Ten(17.5\%) of 57 infants who were on NCPAP initially needed MV during their first 3-days. There wasn't any case with air leak. The incidence of pulmonary hemorrhage, PDA, NEC, İVH, BPD, ROP and mortality were $6.2 \%, 26.2 \%$, $20 \%, 13.8 \%, 10 \%, 10 \%$ and $38.7 \%$, respectively. The duration of respiratory support was 1-720 hours(median: 29 hours). Mean duration of hospitalization was $34.9 \pm 28.4$ days.

Conclusion Our study demonstrated that NCPAP is an effective non-invasive ventilatory strategy. It didn't increase the risk of air leak. The incidences of BPD and ROP in our series were lower than reported in 'NICHD Neonatal Research Network' data.

\section{RESPIRATORY SIGNALS FROM THE EXTERNAL BODY SURFACE IN SPONTANEOUSLY BREATHING PRETERM INFANTS}

doi:10.1136/archdischild-2012-302724.1799

1.2AW Flemmer, ${ }^{2} \mathrm{~L}$ Owen, ${ }^{1} \mathrm{~A}$ Schulze, 'S Herber-Jonat, ${ }^{2} \mathrm{PG}$ Davis. 'Div. Neonatology, Perinatal Center, Ludwig-Maximilian-University Munich, Munich, Germany; ${ }^{2}$ Neonatal Research, Royal Women's Hospital, Melbourne, VIC, Australia

Background Synchronized noninvasive ventilation (sNIPPV) might be superior to fixed time-cycled modes. Several body-surface respiratory signals have been suggested for noninvasive synchronization. However, the quality and utility of such signals have not been evaluated comprehensively.

Methods Respiratory signals in spontaneously breathing preterm infants ( $n=9$ ) were recorded simultaneously (i) respiratory impedance plethysmography (RIPabd \& RIPchest), (ii) Graseby Capsule (GC), (iii) a strapless piezo sensor (Piezo), and (iv) a fluid-filled esophageal pressure catheter (Pes). A total of 5813 breaths were evaluated. Subsequently, periods of good-quality signals on all recorded channels were analyzed with regard to signal availability, time lag relative to Pes, and variability of signals.

Results In all breaths studied $38 \%$ of breaths showed valid signals in all channels without movement artefacts. Of all signals, RIPabd indicated the onset of inspiration earliest in time relative to the onset of inspiration detected by Pes $(-52 \pm 160 \mathrm{~ms})$. RIPabd-signal was followed by GC-signal $(+10 \pm 177 \mathrm{~ms})$. Both signals had a reasonable variability. The Piezo-signal was very sensitive and prone to large variations $(+70 \pm 1372 \mathrm{~ms}$ compared to Pes). RIPchest indicated inspiration later than RIPabd (+104 $\pm 212 \mathrm{~ms})$.

Conclusion These data indicate that both RIPabd and Graseby Capsule are suitable surface sensors for non-invasive synchronization of NIPPV whereas a Piezo sensor exhibited large variability. Signals from all studied sensors were only suitable for a limited amount of time.

\section{SURVEY OF THE CURRENT USE OF N-IPPV IN SPANISH NEONATAL UNITS}

doi:10.1136/archdischild-2012-302724.1800

C Carrasco Carrasco, Respiratory PathologySurfactant Group of the Spanish Society of Neonatology (Respisurf). Neonatology Unit, Sant Joan de Deu Hospital, Esplugues de Llobregat, Spain

Background nIPPV is widely spread in Spanish neonatal units. Little evidence about the best mode of delivery or the appropriate ventilatory parameters of nIPPV is available.

The Aim of this study was to assess the current use of nIPPV in Spanish neonatal units.

Methods A survey was designed and sent by email to neonatal units. The survey collected information about the devices and the ventilatory parameters used to deliver nIPPV over 2010. The use of synchronisation was also interrogated.

Results 87 out of 115 questionnaires were answered and returned (75.6\%). 71 units used nIPPV (81.6\%). Infant Flow ${ }^{\circledR}$ was the most used device $(48 / 71 ; 67.6 \%)$, followed by conventional ventilators (38/71, 53.5\%). The initial ventilatory parameters depended on the device that was used. When Infant Flow ${ }^{\circledR}$ was used, PIPs were set between 8 and $10 \mathrm{cmH}_{2} \mathrm{O}$, whereas when a conventional ventilator was used, PIPs varied between 8 and $18 \mathrm{cmH}_{2} \mathrm{O}$. In contrast, PEEP, inflation rate and inflation time were set in a more stable range regardless of which device was used. Regarding synchronisation, only $13 / 71$ units $(18.3 \%)$ always used synchronisation, whereas $27 / 71$ units (38\%) only used it in some cases. The pneumatic capsule was most frequently used when synchronisation was provided with a percentage of $52 \%$ (21/40 units).

Conclusion The most used device to apply nIPPV in Spanish neonatal units was a variable flow device. Overall, low PIP and low inflation rates were set at the beginning of this therapy. Synchronisation was scarcely used.

\section{SURVEY OF THE CURRENT USE OF NON INVASIVE VENTILATION IN SPANISH NEONATAL UNITS}

doi:10.1136/archdischild-2012-302724.1801

C Carrasco Carrasco, Respiratory Pathology and Surfactant Group of the Spanish Society of Neonatology (Respisurf). Neonatology Unit, Sant Joan de Deu Hospital, Esplugues de Llobregat, Spain

Background Nasal ways of ventilation are being used to minimize lung damage due to endotracheal ventilation. Both nCPAP and nIPPV are progressively being more used in neonatal units, in spite of the lack of evidence about the best mode of delivery of nasal ventilation or the appropiate ventilatory parameters.

The Aim of this study was to assess the current state of the application of nCPAP and nIPPV in Spanish neonatal units. 